\title{
Análisis correlacional de competencias matemáticas de pruebas estandarizadas y pre-requisitos matemáticos en estudiantes de nuevo ingreso a Ingeniería en Computación
}

Correlational analysis of mathematical competences of standardized tests and mathematical prerequisites in students of new entrance to Computer

Engineering

Análise de correlação de competências matemáticas de testes padronizados $e$ pré-requisitos matemáticos em novos alunos para engenheiro de informática

Magally Martínez Reyes Universidad Autónoma del Estado de México, Centro Universitario UAEM Valle de Chalco, México mmreyes@hotmail.com

Anabelem Soberanes-Martín Universidad Autónoma del Estado de México, Centro Universitario UAEM Valle de Chalco, México asoberanesm@uaemex.mx

Juan Manuel Sánchez Soto Universidad Autónoma del Estado de México, Centro Universitario UAEM Valle de Chalco, México sotojmss@yahoo.com.mx

\section{Resumen}

El presente trabajo muestra los datos de correlación acerca del razonamiento verbal, lógico matemático, pensamiento analítico y pensamiento funcional, consideradas como competencias matemáticas en pruebas estandarizadas, con respecto al conocimiento matemático inicial que marcan los programas de estudio para alumnos de nuevo ingreso a la carrera de Ingeniería en Computación del Centro Universitario Valle de Chalco, de la Universidad Autónoma del Estado de México, en el 2017, provenientes de diferentes instituciones educativas de nivel medio superior del Estado de México. Se tomaron los 


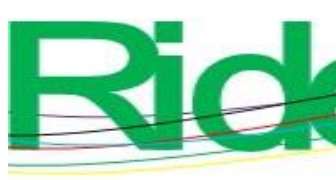

Revista lberoamericana para la

Investigación y el Desarrollo Educativo

ISSN 2007 - 7467

resultados del examen de ingreso a licenciatura (EXANI II) del CENEVAL, de Planea para la localidad de Valle de Chalco y de PISA, y se correlacionaron con un instrumento diagnóstico de 29 ítems que incluye los prerrequisitos que marcan los programas de estudio de las materias de primer semestre del área de matemáticas; se evaluaron conocimientos básicos de aritmética, álgebra, geometría analítica y cálculo diferencial; se consideró a $50 \%$ de la población de alumnos de ingeniería. Existe una diferencia significativa entre los conocimientos en matemáticas que se requieren para ingreso a ingeniería y los contenidos en el EXANI, valorados por medio de una correlación de Pearson y la comparación de medias por $\mathrm{t}$ de student. En todos los instrumentos se obtuvo un bajo nivel operativo de los estudiantes en el área de matemáticas, lo cual indica que las estrategias aplicadas en la trayectoria académica del alumno hasta el nivel medio superior no han favorecido su desarrollo; en ese sentido, se recomiendan actividades didácticas empleando tecnología diseñadas con la intención de favorecer el aprendizaje significativo y conceptual, para fomentar un desempeño más acorde con las características de la carrera profesional.

Palabras clave: competencias, estudiante universitario, ingeniería, matemáticas.

\section{Abstract}

The present work shows the correlation data about verbal reasoning, mathematical logic, analytical thinking and functional thinking, considered as mathematical competences in standardized tests, with respect to the initial mathematical knowledge that mark the curricula for students entering the race again of Engineering in Computing of the Centro Universitario Valle de Chalco, of the Universidad Autónoma del Estado de México, in 2017, coming from different educational institutions of middle level superior of the State of Mexico. The results of the CENEVAL undergraduate entrance exam, from PLANEA for Valle de Chalco and from PISA, were taken and correlated with a 29-item diagnostic tool that includes the prerequisites of the curricula of the subjects of the first semester of the area of mathematics; basic knowledge of arithmetic, algebra, analytical geometry and differential calculus was evaluated; $50 \%$ of the population of engineering students was considered. There is a significant difference between the knowledge in mathematics required for entrance to engineering and the contents in the EXANI, assessed by means of a Pearson correlation and a comparison of means by $\mathrm{t}$ student. In all the instruments a low operational level of the students in the area of mathematics is obtained, which indicates that the strategies applied in 

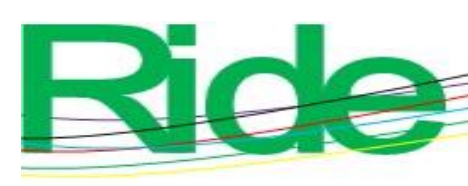

Revista Iberoamericana para la

Investigación y el Desarrollo Educativo

ISSN 2007 - 7467

the academic trajectory of the student to the average superior level have not favored its development; in this sense, it is recommended to use didactic activities using technology designed with the intention of favoring meaningful and conceptual learning, and thus to promote a performance more in line with the characteristics of the professional degree.

Keywords: competencies, university student, engineering, mathematics.

\section{Resumo}

Este artigo mostra os dados de correlação sobre raciocínio verbal, lógica matemática, pensamento analítico e pensamento funcional, considerados como competências matemáticas em testes padronizados, em relação ao conhecimento matemático inicial que marca os programas de estudo para novos alunos que entram na corrida de Engenharia Informática do Centro Universitário Valle de Chalco, da Universidade Autônoma do Estado do México, em 2017, de diferentes instituições de ensino superior do Estado do México. Foram realizados os resultados do exame de admissão ao curso de graduação (EXANI II) do CENEVAL, do Plano para a localidade de Valle de Chalco e do PISA e correlacionados com um instrumento de diagnóstico de 29 itens que inclui os pré-requisitos que marcam os programas de estudo dos assuntos do primeiro semestre da área de matemática; conhecimentos básicos de aritmética, álgebra, geometria analítica e cálculo diferencial; Foram considerados $50 \%$ da população de estudantes de engenharia. Existe uma diferença significativa entre o conhecimento em matemática exigido para a entrada na engenharia e os conteúdos na EXANI, avaliado por meio de uma correlação de Pearson e a comparação dos meios por estudante t. Em todos os instrumentos, obteve-se um baixo nível de operação do aluno na área de matemática, o que indica que as estratégias aplicadas na trajetória acadêmica do aluno até o ensino médio não favoreceram seu desenvolvimento; Nesse sentido, as atividades didáticas são recomendadas usando a tecnologia projetada com a intenção de favorecer a aprendizagem significativa e conceitual, para encorajar uma performance mais adequada às características da carreira profissional.

Palavras-chave: competências, estudantes universitários, engenharia, matemática.

Fecha Recepción: Enero 2017 Fecha Aceptación: Julio 2017 


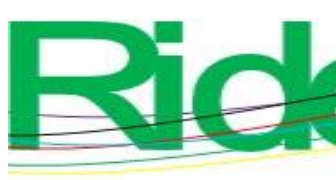

Revista Iberoamericana para la

Investigación y el Desarrollo Educativo

ISSN $2007-7467$

\section{Introducción}

Uno de los principales objetivos de la educación es formar estudiantes autónomos que sean capaces de dirigir su propia experiencia para generar aprendizaje, pero la realidad está generando una paradoja, en la cual el alumno se queja de que las clases son poco interesantes y alentadoras y, por la parte contraria, el profesor se queja de falta de interés y conocimientos del estudiante (Narváez, 2005). En enero del 2008 la Subsecretaria de Educación Media Superior de la Secretaria de Educación Pública de México publicó los documentos “Competencias genéricas y Perfil del Egresado de la Educación Media Superior”, en que establecen las características que debe tener un egresado del sistema medio, indicando que deben de contar con una serie de "competencias que contribuyan al desarrollo de su capacidad de desplegar su potencial, tanto para su desarrollo personal como para el de la sociedad", por lo cual es indispensable tener un enfoque constructivista con base en las competencias (SEP, 2008).

Esta política educativa que guía la enseñanza media superior se topa con resultados poco alentadores cuando se ven evaluados los conocimientos de los estudiantes de dicho nivel en pruebas estandarizadas a nivel nacional e internacional, como es el caso de los recientes resultados de la prueba Planea (2017), que como lo expresa la SEP "son estándares de exigencia más elevados que las pruebas anteriores, en correspondencia con las metas que se ha propuesto la nueva Reforma Educativa en México" (Instituto Nacional de Evaluación Educativa [INEE], 2017). El INEE indica que estos resultados no son comparables con las pruebas Planea 2015 y Planea 2016. Si bien PLANEA 2017 es, como las dos anteriores, una prueba estandarizada, con reactivos de opción múltiple dirigidos a los alumnos que cursan el último semestre del bachillerato en la modalidad escolarizada, este instrumento introdujo una nueva escala de calificación y nuevos puntos de corte para categorizar los resultados en cuatro niveles de desempeño más estrictos que los utilizados anteriormente, obteniendo nuevamente resultados por debajo de los esperados. De tal manera, la prueba explora un mayor número de áreas de conocimiento que en el pasado y sus esquemas de ensamble, aplicación y calificación también se transformaron. En Lenguaje y Comunicación, el promedio nacional alcanzado en 2015 por los alumnos evaluados fue de 496 puntos, mientras que en 2017 fue de 500; es decir, se registró una mejora de 4 puntos (la escala es de 200 a 800 con una media de 500 puntos). 


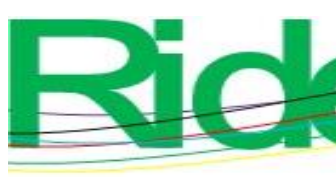

Revista Iberoamericana para la

Investigación y el Desarrollo Educativo

ISSN 2007 - 7467

Asimismo, para el caso de Matemáticas, el puntaje promedio en 2015 fue de 498 y en 2017 de 500 puntos, con un aumento de dos puntos (INEE, 2017).

Los datos de la prueba PLANEA 2017 muestran que los alumnos de educación media superior en el campo de la Comunicación y el Lenguaje se distribuyen de manera homogénea en los tres primeros niveles de logro (33.9\% en el nivel I, $28.1 \%$ en el nivel II, $28.7 \%$ en el nivel III y $9.2 \%$ en el nivel IV); mientras que esto no ocurre en matemáticas, en la que cerca de las dos terceras partes de los estudiantes se concentran en el nivel más bajo del desempeño. Esto indica un problema generalizado de bajos niveles de aprendizaje, de acuerdo con los estándares de la prueba (INEE, 2017).

Algo similar sucede con la evaluación de PISA 2015, donde el desempeño de México se encuentra por debajo del promedio de los países miembros de la Organización para la Cooperación y el Desarrollo Económicos (OCDE) en ciencias (416 puntos), lectura (423 puntos) y matemáticas (408 puntos). En estas tres áreas, menos de 1\% de los estudiantes en México logran alcanzar niveles de competencia de excelencia (nivel 5 y 6). En promedio, el rendimiento en matemáticas mejoró en 5 puntos por cada tres años entre el 2003 y el 2015, pero el promedio del 2015 está por debajo al obtenido el 2009 (419 puntos). Los estudiantes en México declaran altos niveles de interés en ciencias, que se identifica a través de sus expectativas de tener una carrera profesional de esta área, en la importancia de la investigación científica, o de su motivación por aprender ciencias; sin embargo, estas actitudes positivas están débilmente asociadas con el desempeño de los estudiantes en matemáticas (OCDE, 2015).

Por otro lado, el informe Panorama de la Educación 2017 de la OCDE que presenta cifras hasta 2016, muestra que la proporción de adultos jóvenes que finalizaron sus estudios de educación media superior aumentó de $20 \%$ a $25 \%$, y la proporción que terminó la educación superior se incrementó de $17 \%$ a $22 \%$. Lo que indica que la política de elevar a obligatorio los estudios a nivel medio superior decretada en el ciclo escolar 2012-2013 ocasionó que el Estado adquiriera la obligación de "ofrecer un lugar para cursar a quien teniendo la edad típica hubiera concluido la educación básica” (INEE, 2015), que originó un mayor incremento en cobertura e inclusión, ya que se brindó atención educativa a casi 5.5 millones de alumnos, con lo cual se logró una tasa de cobertura total de $82 \%$. Tan solo en la modalidad escolarizada, en los últimos cuatro ciclos escolares la cobertura se incrementó 10.7 puntos 


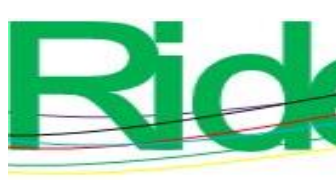

Revista Iberoamericana para la

Investigación y el Desarrollo Educativo

ISSN 2007 - 7467

porcentuales, pasando de $65.9 \%$ a 76.6\%. Por su parte, en educación superior, entre el 2012 y el ciclo 2016-2017, el incremento en la cobertura total fue 5.2 puntos porcentuales, de $32.1 \%$ a 37.3\%; así como se incentivaron carreras del área de tecnológica e ingeniería (OCDE, 2017).

Según la OCDE, "se considera que la Educación Terciaria desempeña un papel esencial en el fomento del conocimiento y la innovación, ambas fundamentales para mantener el crecimiento económico. Varios gobiernos de los países de la OCDE han puesto especial énfasis en mejorar la calidad de la educación en ciencia, tecnología, ingeniería y matemáticas, lo que refleja la importancia de estas disciplinas para impulsar el progreso económico, apoyar la innovación y sentar las bases de una verdadera prosperidad. Además, las habilidades y competencias científicas avanzadas tales como el razonamiento crítico, la resolución de problemas y la creatividad, se consideran fundamentales para tener éxito en el mercado laboral, independientemente de la ocupación final de los estudiantes" (OCDE, 2017). Aunque cada institución por país determina los mecanismos de ingreso al nivel universitario, la mayoría coincide en contemplar los resultados de un examen general de ingreso y el expediente académico del aspirante de nivel medio superior.

En la Universidad Autónoma del Estado de México el mecanismo de ingreso al nivel superior es el EXANI II, una prueba de aptitud académica que evalúa las habilidades intelectuales y los conocimientos específicos, considerados básicos e imprescindibles para iniciar estudios de educación superior. Se aplica a aspirantes a ingresar al nivel de educación superior en instituciones que han contratado los servicios del CENEVAL, y no se aplica a solicitantes individuales. Por el propósito para el que se diseñó el instrumento, aproximadamente 50\% de sus contenidos evalúan habilidades intelectuales (SEP, 2017). Derivadas de las competencias que se buscan desarrollar en estudiantes de la carrera en Ingeniería en Computación, interesan los aspectos relacionados con matemáticas. En un primer momento, ya que la forma de leer e interpretar las preguntas del área de matemáticas requieren de procesos de traducción, interpretación y solución. Mientras en el segundo momento, corresponde a conocimientos y habilidades que son de especial interés para las ingenierías, por lo que se incluye: pensamiento racional, pensamiento analítico, pensamiento funcional y dos módulos específicos de matemáticas y física. 


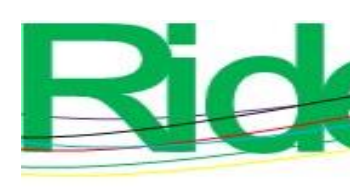

Revista Iberoamericana para la

Investigación y el Desarrollo Educativo

ISSN 2007 - 7467

De manera que se analizaran los resultados relacionados con estas pruebas: Planea, PISA y EXANI II, relacionados con estudiantes de nuevo ingreso a la carrera de Ingeniería en computación, y posteriormente se revisaran los requisitos específicos que marcan los planes de estudio de las asignaturas de matemáticas del primer semestre: Geometría Analítica, Álgebra, Probabilidad y Estadística y Cálculo diferencial e integral, para correlacionar resultados.

\section{Desarrollo}

El proceso de aprendizaje dependerá de cada uno como individuo y de los procesos propios para centrar, procesar, internalizar y recordar información para desarrollar habilidades académicas nuevas; esta dinámica dependerá del tipo de enseñanza que se determine, en donde el alumno puede ser un ente pasivo o activo y enfrentarse a retos cognitivos (Aparicio, 2004; García, 2002; Lejter, 2000; Bruno, 2006). Hay un gran número de referencias, desde hace un par de décadas, que indican que el aprendizaje que se busca en el alumno es de tipo constructivista, que aprenda a partir de sus propias experiencias (Padilla, 2003; Aristegui, 1999) resolviendo sus propios problemas y enfrentar diferentes retos a través de sus capacidades para logran un tipo de aprendizaje que tenga significado en el proceso de desarrollo cognitivo, y se relacione con otros factores biopsicosociales particulares del individuo (Narváez, 2005; Padilla, 2006; Viera, 2003).

\section{PISA}

Esta preocupación por las competencias de los estudiantes plasmada en la enseñanza de las matemáticas tiene muchos años y es una problemática a nivel internacional; de hecho, PISA desde 2007 define la competencia matemática como "la capacidad de un individuo de identificar y comprender el papel de las Matemáticas en el mundo actual, emitir juicios bien fundamentados y utilizarlas y comprometerse con ellas de manera que puedan satisfacer las necesidades de la vida del sujeto como ciudadano constructivo, comprometido y reflexivo" (INEE, 2012). La competencia matemática de PISA no se reduce al dominio de la terminología, los datos y los procedimientos matemáticos ni a la habilidad para realizar diversas operaciones y poner en práctica determinados métodos; la competencia matemática supone una combinación de estos elementos con objeto de responder a exigencias que se plantean en contextos reales. Implica poseer la habilidad para plantear, formular e interpretar 

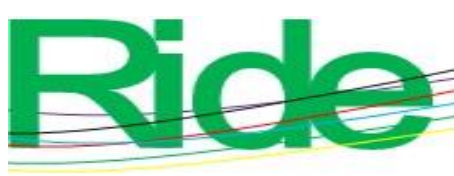

Revista Iberoamericana para la

Investigación y el Desarrollo Educativo

ISSN 2007 - 7467

problemas mediante las matemáticas en una variedad de situaciones y contextos que van desde lo sencillo a lo complejo (INEE, 2012).

De ahí que las pruebas estandarizadas internacionales, como PISA, busquen con la evaluación determinar si los estudiantes pueden reproducir lo que han aprendido, sino que además examina cómo pueden extrapolar lo que han aprendido y aplicar ese conocimiento en circunstancias desconocidas, tanto dentro como fuera de la escuela; es decir, si adquieren la competencia matemática. En México, este concepto se ha manejado como competencia y definido como "un sistema de acción complejo que abarca las habilidades intelectuales, las actitudes y otros elementos no cognitivos, como motivación y valores, que son adquiridos y desarrollados por los individuos a lo largo de su vida y son indispensables para participar eficazmente en diversos contextos sociales" (INEE, 2012). Este enfoque refleja el hecho de que las economías modernas recompensan a los individuos no por lo que saben, sino por lo que pueden hacer con lo que saben. Las conclusiones de los estudios de PISA (2015) permiten a los responsables políticos de todo el mundo medir el conocimiento y las competencias de los estudiantes en sus propios países en comparación con estudiantes de otros países, fijar metas de política educativa en referencia a objetivos medibles conseguidos por otros sistemas educativos y aprender de las políticas y prácticas aplicadas en otros lugares. Los procesos que el estudiante debe realizar corresponden con tres grados de complejidad:

1. En los procesos que PISA llama de reproducción se trabaja con operaciones comunes, cálculos simples y problemas propios del entorno inmediato y la rutina cotidiana.

2. Los procesos de conexión involucran ideas y procedimientos matemáticos para la solución de problemas que ya no pueden definirse como ordinarios pero que aún incluyen escenarios familiares; además involucran la elaboración de modelos para la solución de problemas.

3. El tercer tipo de procesos, los de reflexión, implican la solución de problemas complejos y el desarrollo de una aproximación matemática original. Para ello los estudiantes deben matematizar o conceptualizar las situaciones. 


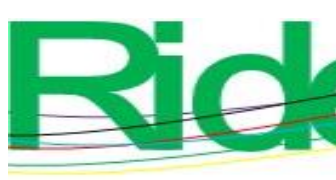

Revista Iberoamericana para la

Investigación y el Desarrollo Educativo

ISSN 2007 - 7467

En estos procesos, según lo formula el INEE, se requiere que los estudiantes "reconozcan y extraigan las matemáticas contenidas en la situación" (OCDE, 2009). Los contenidos de la evaluación de competencia matemática abarcan problemas de cantidad, espacio y forma, cambio y relaciones y probabilidad. Los problemas matemáticos que se plantean están situados en diferentes contextos o situaciones. En este caso se trata de cuatro diferentes situaciones: situación personal, relacionada con el contexto inmediato de los alumnos y sus actividades diarias; situación educativa o laboral, relacionada con la escuela o el entorno de trabajo; situación pública, relacionada con la comunidad; la situación científica, que implica el análisis de procesos tecnológicos o situaciones específicamente matemáticas (OCDE, 2009).

Para efectuar la evaluación en el área de matemáticas se han establecido seis niveles de competencia tanto en la escala combinada como en las subescalas que se refieren a los componentes particulares: cantidad, espacio y forma, cambio y relaciones probabilidad. Los niveles de la escala combinada se definen como sigue:

- Nivel 6 (más de 668 puntos). Los estudiantes que alcanzan este nivel son capaces de conceptualizar, generalizar y utilizar información basada en sus investigaciones y en su elaboración de modelos para resolver problemas complejos. Pueden relacionar diferentes fuentes de información. Demuestran pensamiento y razonamiento matemático avanzado. Pueden aplicar sus conocimientos y destrezas en matemáticas para enfrentar situaciones novedosas. Pueden formular y comunicar con precisión sus acciones y reflexiones.

- Nivel 5 (de 607 a 668 puntos). En este nivel los estudiantes pueden desarrollar y trabajar con modelos para situaciones complejas. Pueden seleccionar, comparar y evaluar estrategias adecuadas de solución de problemas complejos relacionados con estos modelos. Pueden trabajar de manera estratégica al usar ampliamente habilidades de razonamiento bien desarrolladas, representaciones de asociación y caracterizaciones simbólicas y formales.

- Nivel 4 (de 545 a 606 puntos). Los estudiantes son capaces de trabajar efectivamente con modelos explícitos para situaciones complejas concretas. Pueden seleccionar e integrar diferentes representaciones, incluyendo símbolos y asociándolos directamente 


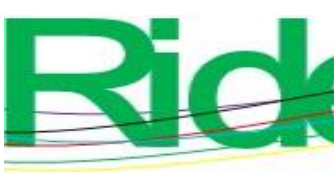

Revista Iberoamericana para la

Investigación y el Desarrollo Educativo

ISSN 2007 - 7467

a situaciones del mundo real. Pueden usar habilidades bien desarrolladas y razonar flexiblemente con cierta comprensión en estos contextos. Pueden construir y comunicar explicaciones y argumentos.

- Nivel 3 (de 483 a 544 puntos). Quienes se sitúan en este nivel son capaces de ejecutar procedimientos descritos claramente, incluyendo aquellos que requieren decisiones secuenciales. Pueden seleccionar y aplicar estrategias simples de solución de problemas. Pueden interpretar y usar representaciones basadas en diferentes fuentes de información, así como razonar directamente a partir de ellas. Pueden generar comunicaciones breves para reportar sus interpretaciones.

- Nivel 2 (de 421 a 482 puntos). En el segundo nivel los alumnos pueden interpretar y reconocer situaciones en contextos que requieren únicamente de inferencias directas. Pueden extraer información relevante de una sola fuente y hacer uso de un solo tipo de representación. Pueden emplear algoritmos, fórmulas, convenciones o procedimientos básicos. Son capaces de hacer interpretaciones literales de los resultados.

- Nivel 1 (de 358 a 420 puntos). Los estudiantes son capaces de contestar preguntas que impliquen contextos familiares donde toda la información relevante esté presente y las preguntas estén claramente definidas. Son capaces de identificar información y desarrollar procedimientos rutinarios conforme a instrucciones directas en situaciones explícitas. Pueden llevar a cabo acciones que sean obvias y seguirlas inmediatamente a partir de un estímulo.

- Por debajo del nivel 1 (menos de 358 puntos). Se trata de estudiantes que no son capaces de realizar las tareas de matemáticas más elementales que pide PISA.

En los reportes de PISA, en promedio en los países OCDE, casi uno de cada cuatro estudiantes (23\%) no al alcanza el nivel básico de competencia (Nivel 2) en matemáticas. Los estudiantes que no alcanzan este nivel pueden de vez en cuando realizar procedimientos rutinarios, tales como operaciones aritméticas en situaciones donde todas las instrucciones se les son dadas, pero tienen problemas identificando cómo una (simple) situación del mundo real puede ser representada matemáticamente (por ejemplo, comparar la distancia total entre dos rutas alternativas, o convertir precios a una moneda diferente). La proporción de 


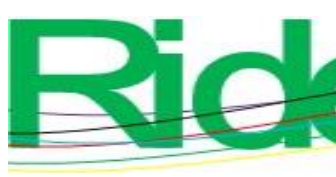

Revista Iberoamericana para la

Investigación y el Desarrollo Educativo

ISSN 2007 - 7467

estudiantes mexicanos que no alcanzan el nivel mínimo de competencia permaneció estable entre el 2003 y el 2015 (PISA, 2015). En este informe de 2015, la evaluación se centra en las materias escolares básicas de ciencia, lectura y matemáticas. También se evalúan las capacidades de los alumnos en un ámbito innovador (en 2015, ese ámbito fue la resolución colaborativa de problemas).

\section{PLANEA}

Inscrita en el Plan Nacional de Evaluación de los Aprendizajes diseñado por el INEE, la prueba Planea Media Superior (Planea MS) 2016 tiene el propósito de informar a la sociedad sobre el estado que guarda la educación, en términos de logro de aprendizaje de los estudiantes, en dos áreas de competencia: Lenguaje y Comunicación (Comprensión Lectora) y Matemáticas. La prueba está dirigida a los alumnos de toda la República Mexicana inscritos en los planteles que manifiesten interés en participar en la aplicación de Planea MS con una periodicidad anual. Los estudiantes deben cursar su último ciclo de bachillerato (cuatrimestre, semestre, año, etcétera) en modalidad escolarizada, en los diferentes subsistemas y modalidades de Escuela Media Superior (EMS). Las instituciones pueden ser tanto de sostenimiento público como privado. Es una evaluación diagnóstica individual que consta de 110 reactivos de opción múltiple, cuyo resultado se utiliza con fines de retroalimentación y permite conocer el nivel de dominio que alcanza el sustentante en indicadores de competencia asociados a las áreas que evalúa la prueba. Planea MS es una prueba que se enfoca a un conjunto limitado de conocimientos y habilidades que un grupo de expertos considera indicadores suficientes y representativos de las competencias disciplinares básicas que, idealmente, deben dominar los sustentantes (INEE, 2016).

El enfoque pedagógico por competencias reconoce que a la solución de cada tipo de problema matemático corresponden diferentes conocimientos y habilidades, y el despliegue de diferentes valores y actitudes. Por ello, los estudiantes deben razonar matemáticamente, y no simplemente responder ciertos tipos de problemas mediante la repetición de procedimientos establecidos. Esto implica que puedan llevar las aplicaciones de esta disciplina más allá del salón de clases. De las ocho competencias del Modelo Centrado en Competencias para matemáticas, se eligieron las siguientes seis: 
- Interpreta modelos matemáticos mediante la aplicación de procedimientos aritméticos, algebraicos, geométricos y variacionales, para la comprensión y análisis de situaciones reales, hipotéticas o formales.

- Resuelve problemas matemáticos, aplicando diferentes enfoques.

- Interpreta los datos obtenidos mediante procedimientos matemáticos y los contrasta con modelos establecidos o situaciones reales.

- Analiza las relaciones entre dos o más variables de un proceso social o natural para determinar o aproximar su comportamiento.

- Cuantifica y representa matemáticamente las magnitudes del espacio y las propiedades físicas de los objetos que lo rodean.

- Lee tablas, gráficas, mapas, diagramas y textos con símbolos matemáticos y científicos.

La prueba de Matemáticas de Planea MS evalúa la capacidad de un individuo para identificar, interpretar, aplicar, sintetizar y evaluar matemáticamente su entorno, haciendo uso de su creatividad y de un pensamiento lógico y crítico que le permita solucionar problemas cuantitativos con diferentes herramientas matemáticas. La capacidad matemática que evalúa la prueba se cristaliza en reactivos asociados a contenidos aritméticos, geométricos y algebraicos que se consideran los mínimos indispensables para los sustentantes al terminar el bachillerato y que se categorizan en: cantidad, espacio y forma y cambios y relaciones, con los procesos cognitivos de reproducción, conexión y reflexión. Estas categorías son semejantes a las de PISA. Se establecen cuatro niveles de dominio (CENEVAL, 2016):

1. Los alumnos que se encuentran en este nivel de logro demuestran deficiencias en el desarrollo de los conocimientos y habilidades relacionados con las competencias disciplinares básicas que se esperan de los egresados de la educación media superior; además, todavía presentan dificultad para realizar las tareas que se indican en los niveles 2, 3 y 4, ya que solo muestran habilidad para resolver problemas directos que requieren efectuar operaciones básicas con números enteros e identificar elementos gráficos. 
2. Los alumnos que se encuentran en este nivel de logro son capaces de aplicar procedimientos aritméticos y geométricos simples para la comprensión de diversas situaciones similares a las que se estudian en el aula, además de la identificación de relaciones espaciales. Realizan operaciones con fracciones, porcentajes o con signos de agrupación; representan gráficamente series de números, o describen el comportamiento de sucesiones numéricas y la relación entre ellas. Transforman modelos matemáticos de naturaleza algebraica o geométrica cuando enuncian en lenguaje común una expresión algebraica y viceversa, además de que resuelven problemas geométricos bidimensionales y tridimensionales que involucran transformaciones y el manejo de los elementos de las figuras. Resuelven sistemas de ecuaciones e identifican la combinación de procedimientos necesarios para solucionar diferentes ejercicios. Sin embargo, todavía demuestran un dominio deficiente de las tareas que se indican en los niveles 3 y 4 .

3. Además de dominar los conocimientos y habilidades del nivel 2 , los alumnos que se encuentran en este nivel de logro son capaces de analizar las relaciones entre dos o más variables de un problema contextualizado para estimar u obtener un resultado. Resuelven problemas relacionados con procesos sociales o naturales que involucran variables y unidades físicas, y realizan cálculos con razones y proporciones. Resuelven problemas matemáticos aplicando diferentes enfoques, ya sea que requieran del planteamiento de ecuaciones, la aplicación del teorema de Pitágoras o de conceptos como el mínimo común múltiplo y el máximo común divisor, o exijan estimar soluciones para problemas aritméticos, geométricos o variacionales. Además, extraen información de tablas o gráficas para resolver problemas que involucran operaciones. Sin embargo, todavía demuestran un dominio deficiente de las tareas que se indican en el nivel 4.

4. Además de dominar los conocimientos y habilidades de los niveles 1 y 2 , los alumnos que se encuentran en este nivel de logro son capaces de evaluar el entorno e integrar los datos obtenidos mediante diferentes procedimientos matemáticos, para contrastarlos con modelos establecidos o situaciones reales. Leen e interpretan tablas, gráficas e información textual cuando resuelven problemas contextualizados que requieren de estimaciones, conversiones, análisis de información gráfica o sucesiones. 


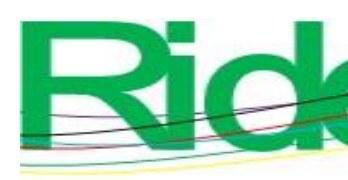

Revista Iberoamericana para la

Investigación y el Desarrollo Educativo

ISSN 2007 - 7467

Cuantifican y representan matemáticamente las magnitudes del espacio para resolver problemas que implican el manejo de figuras planas y tridimensionales, así como las propiedades geométricas de figuras incompletas. Adicionalmente, realizan cálculos a partir de dos funciones lineales o cuadráticas que se muestran de manera independiente y mediante representaciones numéricas, textuales, gráficas o tabulares.

En los resultados 2016 de Planea MS para el municipio de Valle de Chalco, donde pertenecen las instituciones de los sujetos de estudio y parte de la muestra, se tiene que $72 \%$ se encuentra en nivel 1, 20\% en nivel 2, $8 \%$ en nivel 3 y ninguno en nivel 4. Estos resultados concuerdan con los recabados a nivel nacional donde $49.2 \%$ está en nivel 1, 30\% en nivel 2, $14.4 \%$ en nivel 3 y $6.3 \%$ en nivel 4 . Desde su concepción, la prueba es útil para conocer las fortalezas y áreas de oportunidad que exhiben los alumnos en lo que se definió operacionalmente como parte de la evaluación en Matemáticas (CENEVAL, 2016).

\section{EXANI}

En otro contexto diferente se encuentra el EXANI II, una prueba estandarizada de aptitud académica a nivel nacional que evalúa las habilidades intelectuales y los conocimientos básicos e imprescindibles para iniciar estudios de educación superior. Al ser un servicio que las instituciones de educación superior contratan al CENEVAL, los resultados son confidenciales para cada escuela con el fin de establecer el nivel de potencialidad de un individuo para lograr nuevos aprendizajes; aunque se proporciona de manera individual los resultados a cada participante con la intención de que estimen las áreas de oportunidad que deben atacar para un buen desempeño en el ámbito universitario. Para el caso de ingreso a cualquier carrera se consideran, a nivel nacional, las competencias de: Pensamiento matemático (PM), Pensamiento analítico (PA), Estructura del lenguaje (EL), Comprensión lectora (CL), Lenguaje escrito (LE) e Inglés (IN), mientras que para el caso particular de Ingeniería en Computación se agregan dos módulos más: Matemáticas (MOD01) y Física (MOD02). Para efectos de este estudio, nos concentraremos en: Pensamiento matemático, Pensamiento analítico y Matemáticas, para acercarnos a los indicadores de las competencias de los otros instrumentos estandarizados (PISA y Planea). 


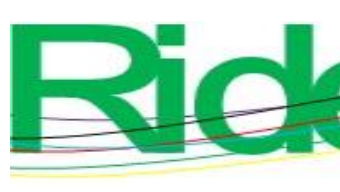

Revista Iberoamericana para la

Investigación y el Desarrollo Educativo

ISSN $2007-7467$

El EXANI II evalúa la habilidad de conocimiento e identificación de información y contenidos específicos; también, la capacidad de sistematización e integración mediante el uso de fórmulas, reglas o teorías, contemplar esquemas o cuadros sinópticos o, bien, la clasificación, ordenamiento o agrupación de información; finalmente, también indaga la competencia de interpretación y aplicación mediante situaciones que exigen encontrar una estrategia apropiada para realizar inferencias, establecer conclusiones y solucionar problemas. En particular, el área de Pensamiento matemático explora la competencia para comprender y resolver situaciones que implican el uso de estrategias de razonamiento aritmético, algebraico, estadístico y probabilístico, y geométrico. Es decir, abarca el conjunto de conocimientos y habilidades del campo matemático que debieron aprenderse y dominarse en la educación media superior (EMS). En el área de Pensamiento analítico el aspirante debe demostrar su competencia a nivel intermedio para integrar y analizar información de tipo textual y gráfica; también debe ser capaz de comprender e interpretar relaciones lógicas y patrones, así como reconocer y analizar las coincidencias en la representación espacial de objetos en diferentes planos. El área de Estructura de la lengua evalúa la capacidad para identificar y aplicar elementos de la lengua que permiten la creación y organización de mensajes con sentido. El área de Comprensión lectora demanda comprender información explícita e implícita en textos narrativos e informativos, así como su propósito, características y lenguaje.

Finalmente, para las áreas de Lenguaje escrito, Inglés, Matemáticas y Física evalúan el nivel de desempeño de los aspirantes para reconocer, comprender y resolver planteamientos en los que debe aplicar los conocimientos y las habilidades adquiridos en las asignaturas de la EMS (CENEVAL, 2017). Es factible observar que lo que PISA y Planea especifica como Competencias Matemáticas, corresponden a lo que el EXANI II está estableciendo como categorías de análisis. El EXANI no establece niveles de desempeño sino puntajes de aciertos para un total de 200 reactivos, distribuidos como sigue: PM (19), PA (67), EL (7), CL (7), LE (30), IN (20), MOD01 (35) y MOD02 (15).

En este contexto, y además enfatizando la necesidad de desarrollar determinadas competencias matemáticas en estudiantes de nivel medio superior, como lo marca la National Council of Teacher of Mathematics (NCTM 1989), se determinan los estadios para llevar a un estudiante de matemáticas de este nivel del pensamiento básico al avanzado, del pensamiento 

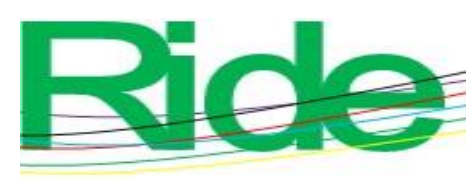

Revista Iberoamericana para la

Investigación y el Desarrollo Educativo

ISSN 2007 - 7467

concreto al abstracto. Al respecto, Cuevas y Pluvinage (2017) establecen los siguientes estadios del pensamiento matemático básico desde el punto de vista cognitivo:

- Pensamiento aritmético, que corresponde al conocimiento de los números y operaciones aritméticas básicas (Kjeldsen y Petersen, 2014; Mouhayar y Jurdak, 2016).

- Pensamiento aritmético avanzado (proporciones), que se refiere a un conocimiento numérico más avanzado; esto es, a partir de las operaciones aritméticas básicas se desarrollan las proporciones y el manejo de fracciones, incluyendo también el conocimiento de magnitudes y elementos de razonamiento lógico (Pluvinage y Cuevas, 2006).

- Pensamiento algebraico, que incluye reconocer y analizar patrones, estudiar y representar relaciones, hacer generalizaciones y analizar cómo cambian las cosas. También contiene los Sistemas Matemáticos de Signos (SMS); la resolución de ecuaciones y el manejo de expresiones que incluyen variables y parámetros. Es conveniente señalar que la adquisición de este pensamiento facilitaría la comprensión del simbolismo abstracto y el trabajo con relaciones algebraicas (Filloy, Puig y Rojano, 2008; Lagrange, 2014; Seeley, 2004).

- Pensamiento funcional, que se define como una actividad cognitiva que permite establecer relaciones de dependencia funcional, más allá de las relaciones aritméticas y algebraicas que pueden ser aplicadas a diversos contextos y que no se relaciona con la aplicación de fórmulas. Aún más, se considera que para poder interactuar en el mundo actual y poder desarrollarse en cualquiera de las diversas disciplinas o ciencias tanto básicas, naturales como sociales, se requiere de un pensamiento funcional (Pluvinage y Cuevas, 2006).

De igual manera, autores, como García (2014), indican que “... la ingeniería es una disciplina de carácter reflexivo-pragmático que tiende a dejar la fundamentación teórica de los recursos de conocimiento que utiliza a ser desarrollada por otras disciplinas, como es el caso de las matemáticas".

De manera que en este amplio contexto y para uniformizar el análisis, se establecerán los cuatro tipos de pensamiento como unidades de análisis a través de un instrumento que incluye 


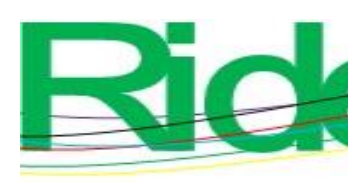

Revista Iberoamericana para la

Investigación y el Desarrollo Educativo

ISSN $2007-7467$

29 reactivos y que fue aplicado a 50\% del total de alumnos de nuevo ingreso a la carrera de Ingeniería en Computación del Centro Universitario UAEM Valle de Chalco; los resultados obtenidos se compararon con los datos de PISA, Planea y EXANI II para su análisis.

\section{Pretest}

Con el fin de evaluar el desempeño escolar en el nivel superior en las materias de primer año universitario: Geometría Analítica, Algebra, Probabilidad y Estadística y Cálculo diferencial e integral, de la carrera de Ingeniería en Computación, se diseñó un instrumento de evaluación para valorar lo que estas asignaturas marcan como prerrequisitos en sus planes de estudio, conformando 29 reactivos divididos conforme a la categorización de los cuatro estratos matemáticos básicos tomados de la clasificación anterior (Cuevas y Pluvinage, 2017): el aritmético, el aritmético avanzado, el algebraico y el funcional. Estos estratos determinan el conocimiento básico (requisitos matemáticos) que un alumno debe dominar considerando su nivel de estudios y su grado de madurez. Para el análisis se asignó a cada pregunta un valor 1 (correcto), 2 (incorrecto), 3 (no contesta) y 4 (otra solución). Este instrumento ha evolucionado a lo largo de varios años de aplicación y retroalimentación desde el 2006 y se ha sometido a pruebas de validez y confiabilidad (Martínez, Soberanes y Castillo, 2016). Los resultados para el grupo tomado como muestra serán presentados enseguida y comparados con los resultados de las pruebas estandarizadas.

\section{Método}

La preocupación por analizar las competencias matemáticas de los alumnos que egresan del nivel medio superior e ingresan al superior es compartida por una amplia comunidad; es posible encontrar en diferentes foros los estudios que distintas instituciones realizan con el fin de tener un punto de partida para la enseñanza universitaria. Por ejemplo, Rico (2007) realiza un análisis interesante de las competencias especificadas en la prueba PISA del 2007 y que se han mantenido y enriquecido a lo largo de los años; por ello, en este estudio se retomaron las competencias especificadas en las tres pruebas estandarizadas PISA, EXANI y Planea, para mostrar su similitud. Sin embargo, no proporcionan un marco de análisis común, por lo que se englobaron en los niveles de Pensamiento Aritmético, Pensamiento Algebraico y Pensamiento Funcional. 


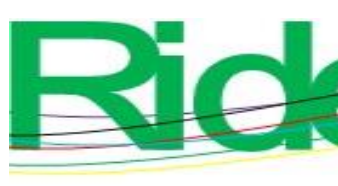

Revista Iberoamericana para la

Investigación y el Desarrollo Educativo

ISSN 2007 - 7467

En el mismo sentido, es generalizada la preocupación por medir las competencias matemáticas mediante los exámenes de ingreso nacionales, que determinan un lugar en las universidades. Por ejemplo, encontramos estudios como los de Larrazolo, Backhoff y Tirado (2013) que miden 45 competencias matemáticas del Examen de Habilidades y Conocimientos Básicos (Exhcoba), utilizado en los procesos de admisión de 2006 y 2007 para las universidades estatales de Querétaro, Nayarit, Sonora, Guanajuato y Baja California, donde los resultados confirman que los estudiantes: tienen un aprovechamiento sumamente bajo; no comprenden los conceptos básicos de matemáticas; no tienen las habilidades para solucionar problemas numéricos de mediana complejidad y los conocimientos adquiridos se relacionan con la memorización de algoritmos. Por ello, en el presente estudio tomamos indicadores adicionales extendiendo este tipo de estudios, relacionando PISA, Planea MS y EXANI II y un pretest diagnóstico del grupo de nuevo ingreso, para correlacionar resultados.

Por su parte, Villalón, Medina y Bravo (2015) realizan un estudio para determinar las competencias matemáticas previas de los estudiantes de diez Ingenierías en el Instituto Tecnológico de Celaya, comparando la demanda de competencias previas deseables en los estudiantes de nuevo ingreso con las competencias que realmente poseen, con la intención de que la información sirva de insumo para medir la distancia media entre un perfil de ingreso más próximo a la realidad y el nivel formativo solicitado por la institución. Los autores elaboran un examen de exploración de 20 preguntas que considera las competencias matemáticas previas necesarias de los aspirantes, aplicado a 200 estudiantes de nivel medio superior quienes buscan ingresar al Instituto Tecnológico de Celaya; el examen se basa en los contenidos propuestos por el Ceneval para el EXANI II que aplican para la selección de aspirantes a ingresar a la institución; los autores encontraron que los alumnos carecen del nivel de competencias matemáticas previas, por lo que propusieron un taller de Precálculo en los niveles medio superior para atacar esta problemática. De manera que, siguiendo esta línea de exploración, en nuestro estudio elaboramos el Pretest con la intención de medir las competencias matemáticas, pero desde el marco conceptual de los niveles de pensamiento como medida para homogenizar entre lo que solicita Planea MS, PISA y EXANI como competencias matemáticas. 

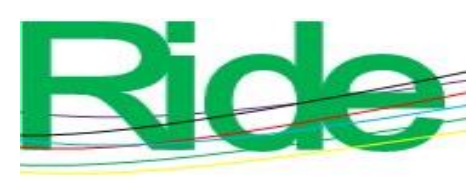

Revista Iberoamericana para la

Investigación y el Desarrollo Educativo

ISSN $2007-7467$

Se tomaron los resultados del EXANI II, examen de ingreso del CENEVAL, de alumnos de nuevo ingreso a la carrera de Ingeniería de Computación del Centro Universitario Valle de Chalco de la Universidad Autónoma del Estado de México para el periodo del 2017. Los alumnos son egresados de diferentes instituciones educativas de EMS del municipio de Valle de Chalco y del área metropolitana, de los cuales se tienen sus resultados de la aplicación de Planea MS 2016. Estos resultados se correlacionaron con el instrumento denominado Pretest, que constó de 29 ítems y que evaluó los conocimientos básicos de aritmética, algebra y relación funcional; las opciones de respuesta para cada ítem se clasificaron en correcta, incorrecta y no contesta u otra solución, se consideró a 50\% de la población de alumnos de Ingeniería en Computación. El cálculo correlacionar se realizó a través del estadígrafo de Pearson para medir el nivel de correlación de cada una de las categorías contempladas en EXANI II y Pretest, para determinar si se está evaluando los mismos contenidos. Además, se realiza una comparación de las medias con una t de student para determinar el nivel de relación de los contenidos de ambas pruebas y de esa manera apoyar las conclusiones sobre la discrepancia o similitud; ambos estadísticos se realizan por medio de software estadístico SPSS.

\section{Resultados}

La tabla 1 representa las calificaciones obtenidas de los egresados del nivel medio superior del examen realizado por CENEVAL, EXANI II, por áreas de competencia: Pensamiento Matemático (PM), Pensamiento Analítico (PA), Estructura del lenguaje (EL) y Matemáticas (MOD01); y resultados del Pretest en sus módulos de: Pensamiento Aritmético (PPA), Pensamiento Algebraico (PPAL) y Pensamiento funcional (PPF), conforme a sus indicadores, en una escala del 1 al 10. 
Tabla 1. Calificaciones del EXANI II.

\begin{tabular}{|lc|c|c|c|} 
& \multicolumn{1}{c}{ Mínimo } & Máximo & Media & $\begin{array}{c}\text { Desviación } \\
\text { típica }\end{array}$ \\
\cline { 2 - 5 } Pensamiento Matemático (PM) & 4.00 & 9.20 & 6.3107 & 1.18123 \\
Pensamiento Analítico (PA) & 4.00 & 9.10 & 6.2733 & 1.22275 \\
\hline $\begin{array}{l}\text { Estructura del Lenguaje (EL) } \\
\text { Matemáticas (MOD01) }\end{array}$ & 2.00 & 8.40 & 4.9000 & 1.52157 \\
\hline Pensamiento Aritmético (PPA) & 3.00 & 8.70 & 5.9767 & 1.29792 \\
\hline Pensamiento Algebraico (PPAL) & 1.43 & 10.00 & 4.7497 & 1.99137 \\
\hline Pensamiento funcional (PPF) & 1.11 & 8.89 & 4.2035 & 1.85630 \\
\hline
\end{tabular}

Fuente: Elaboración propia.

Como se puede observar en el EXANI II, hay una tendencia apenas aprobatoria en PM, PA y MOD01. En PM se incluye razonamiento aritmético con el manejo de las operaciones básicas y números decimales y fraccionarios; relaciones de proporcionalidad; razonamiento algebraico de operaciones con monomios y binomios, ecuaciones de primer y segundo grado, sistemas de ecuaciones y representación gráficas de funciones; razonamiento estadístico y probabilístico y razonamiento geométrico y trigonométrico. En PA se maneja la integración de la información a partir de textos, graficas, mapas, tablas; interpretación de relaciones lógicas; reconocimiento de patrones mediante sucesiones; representación espacial de figuras y objetos. En MOD01 se incluye aritmética mediante problemas con números reales, notación científica y manejo de proporciones en nivel intermedio, de álgebra las ecuaciones lineales y cuadráticas y operaciones con exponentes y radicales, de geometría el manejo de semejanza, áreas, ecuaciones y pendiente, de cálculo corresponde a operaciones con funciones, limites, derivadas e integrales.

Por otro lado, para los contenidos similares que se contemplan en el Pretest se observan valores más bajos: los módulos que contemplan álgebra y aritmética PPA y PPAL están cerca del 5, mientras el de pensamiento funcional PPF no llega a 3 y el pensamiento funcional está muy por debajo de la escala.

Así, para poder relacionar todos los contenidos se establecen en los tres tipos de pensamiento: Aritmético (PPA), Algebraico (PPAL) y Funcional (PPF), y se requiere comparar con los contenidos que está evaluando el EXANI II, lo que produce una primera contradicción en cuanto a escalas de evaluación. Si ahora analizamos el nivel de correlación entre PM, PA, MOD01 con PPA, PPAL y PPF, utilizando correlación de Pearson, se pueden ver los resultados en la Tabla 2. 
Tabla 2. Correlación de Pearson.

\begin{tabular}{|c|c|c|c|c|c|c|c|c|}
\hline \multirow{4}{*}{ PM } & & PM & PA & EL & MOD01 & PPA & PPAL & PPF \\
\hline & $\begin{array}{l}\text { Correlación } \\
\text { de Pearson }\end{array}$ & 1 & $.351^{*}$ & .119 & $.427^{* *}$ & .120 & .041 & $-.310 *$ \\
\hline & $\begin{array}{c}\text { Sig. } \\
\text { (unilateral) }\end{array}$ & & .029 & .265 & .009 & .263 & .414 & .048 \\
\hline & $\mathrm{N}$ & 30 & 30 & 30 & 30 & 30 & 30 & 30 \\
\hline \multirow{3}{*}{ PA } & $\begin{array}{l}\text { Correlación } \\
\text { de Pearson }\end{array}$ & $.351^{*}$ & 1 & .125 & .198 & .064 & .098 & .085 \\
\hline & $\begin{array}{c}\text { Sig. } \\
\text { (unilateral) }\end{array}$ & .029 & & .255 & .148 & .369 & .304 & .327 \\
\hline & $\mathrm{N}$ & 30 & 30 & 30 & 30 & 30 & 30 & 30 \\
\hline \multirow{3}{*}{ EL } & $\begin{array}{l}\text { Correlación } \\
\text { de Pearson }\end{array}$ & .119 & .125 & 1 & -.003 & $.355^{*}$ & .134 & .172 \\
\hline & $\begin{array}{c}\text { Sig. } \\
\text { (unilateral) }\end{array}$ & .265 & .255 & & .493 & .027 & .241 & .182 \\
\hline & $\mathrm{N}$ & 30 & 30 & 30 & 30 & 30 & 30 & 30 \\
\hline \multirow{3}{*}{ MOD01 } & $\begin{array}{l}\text { Correlación } \\
\text { de Pearson }\end{array}$ & $.427^{* * *}$ & .198 & -.003 & 1 & .224 & $-.312^{*}$ & .095 \\
\hline & $\begin{array}{c}\text { Sig. } \\
\text { (unilateral) }\end{array}$ & .009 & .148 & .493 & & .117 & .047 & .308 \\
\hline & $\mathrm{N}$ & 30 & 30 & 30 & 30 & 30 & 30 & 30 \\
\hline \multirow{3}{*}{ PPA } & $\begin{array}{l}\text { Correlación } \\
\text { de Pearson }\end{array}$ & .120 & .064 & $.355^{*}$ & .224 & 1 & .151 & .244 \\
\hline & $\begin{array}{c}\text { Sig. } \\
\text { (unilateral) }\end{array}$ & .263 & .369 & .027 & .117 & & .186 & .073 \\
\hline & $\mathrm{N}$ & 30 & 30 & 30 & 30 & 37 & 37 & 37 \\
\hline \multirow{3}{*}{ PPAL } & $\begin{array}{l}\text { Correlación } \\
\text { de Pearson }\end{array}$ & .041 & .098 & .134 & $-.312^{*}$ & .151 & 1 & .216 \\
\hline & $\begin{array}{c}\text { Sig. } \\
\text { (unilateral) }\end{array}$ & .414 & .304 & .241 & .047 & .186 & & .099 \\
\hline & $\mathrm{N}$ & 30 & 30 & 30 & 30 & 37 & 37 & 37 \\
\hline \multirow{3}{*}{ PPF } & $\begin{array}{l}\text { Correlación } \\
\text { de Pearson }\end{array}$ & $-.310^{*}$ & .085 & .172 & .095 & .244 & .216 & 1 \\
\hline & $\begin{array}{c}\text { Sig. } \\
\text { (unilateral) }\end{array}$ & .048 & .327 & .182 & .308 & .073 & .099 & \\
\hline & $\mathrm{N}$ & 30 & 30 & 30 & 30 & 37 & 37 & 37 \\
\hline
\end{tabular}

* La correlación es significativa al nivel 0,05 (unilateral).

** La correlación es significante al nivel 0,01 (unilateral).

Fuente: Elaboración propia.

La tabla 2 describe las correlaciones de Pearson de cada una de las categorías contempladas en EXANI II y Pretest, en que sobresale la poca relación, a pesar de que explícitamente se describen los contenidos que ambos están evaluando y que confirma la contradicción inicial en cuanto a escalas de evaluación. De manera particular, los únicos módulos de ambas pruebas que tienen una relación significativa son los módulos PM y MOD01 del EXANI II al duplicar el tipo de contenidos que está evaluando, y ambos incluyen aritmética y álgebra, pero 

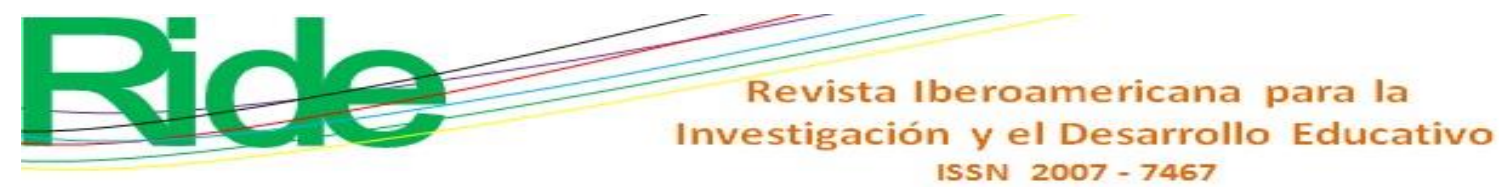

no se correlaciona con ningún modulo del Pretest. Por otro lado, hay una correlación menor pero existente entre PM y MOD01 del EXANI y PPF PPAL del Pretest respectivamente. Estos resultados indican que debe elaborarse un análisis más profundo de los contenidos, para establecer o negar una verdadera relación entre contenidos y calificaciones, lo cual realizaremos con la t de student (ver Tabla 3 ).

Tabla 3. Diferencia relacionada entre el Pretest y el EXANI (categoría PM).

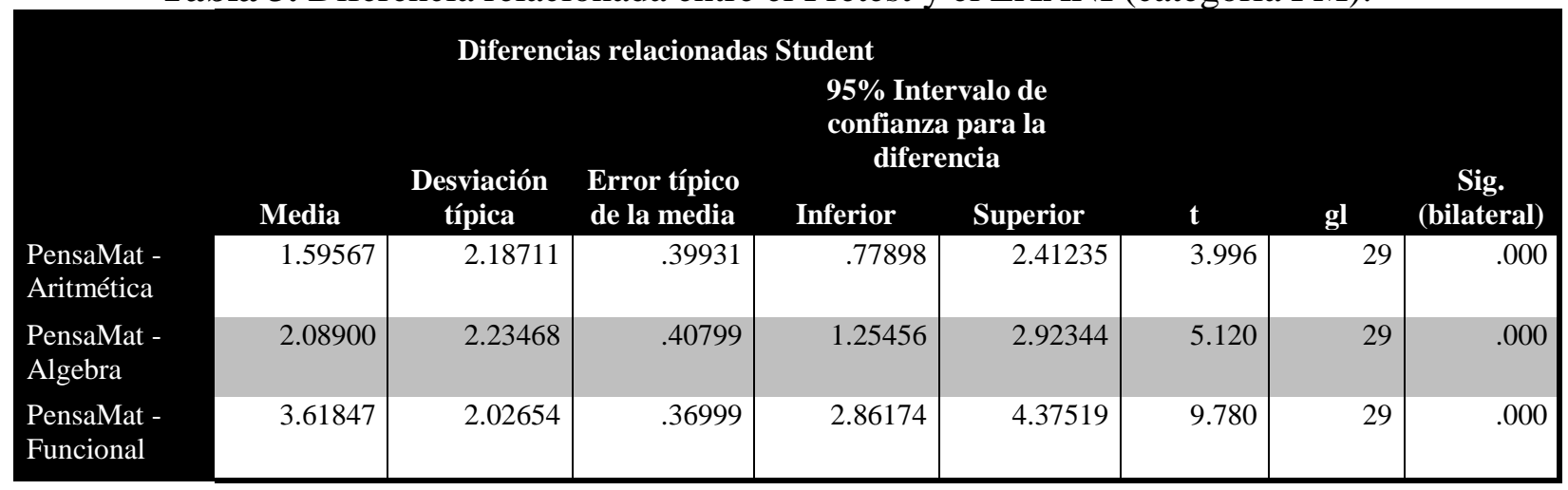

Fuente: Elaboración propia.

Para este análisis se consideró el nivel de similitud de las medias en el intervalo [3 - 4], y de desigualdad en el resto del rango. Se puede observar que solo el PPA guarda relación con el PM, en cuanto a contenidos. En la tabla 4 se presenta el estudio similar para la categoría MOD01.

Tabla 4. Diferencia relacionada entre el Pretest y el EXANI (categoría MOD01).

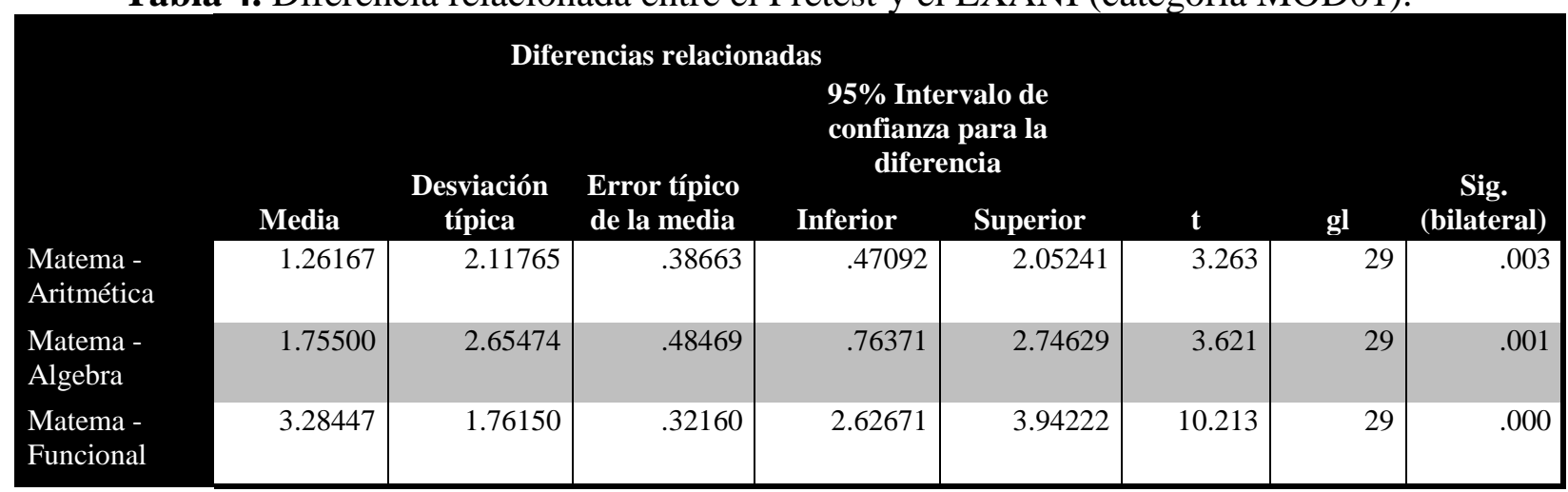

Fuente: Elaboración propia.

Para este análisis se mantuvo el nivel de similitud de las medias en el intervalo [3 - 4], y de desigualdad en el resto del rango. Se puede observar que solo el PPF guarda relación con el MOD01 en cuanto a contenidos. 


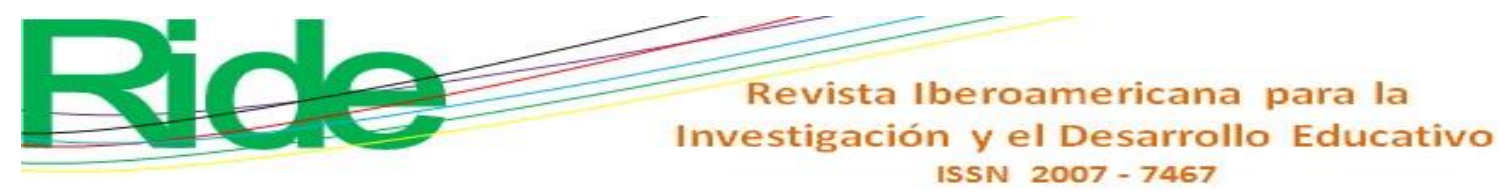

De este ejercicio de análisis entre contenidos específicos que se preguntan en EXANI y Pretest se puede deducir la disparidad de reactivos, aunque pertenecen a los mismos temas evaluados, lo que indica que debe realizarse a mayor profundidad la indagación de si lo que está preguntando el EXANI corresponde a las competencias matemáticas que el mismo instrumento dice evaluar y a las que se requieren de un estudiante de nuevo ingreso de la carrera de Ingeniería en Computación. Además, si se incluyen los otros resultados de PISA y Planea, se vuelve un mar de información que el profesor debe procesar para partir de un cierto nivel de competencia de sus alumnos, seleccionar las estrategias más acordes para que alcancen las competencias que no poseen y lograr los objetivos que las diferentes asignaturas marcan como aprendizajes esperados por los alumnos al concluir un curso de primer año universitario.

Previamente, los estudios presentados por Larrazolo et al. (2013) y Villalón et al. (2015) habían mostrado que los estudiantes de primer año universitario, en particular los de ingenierías, adolecen de las competencias matemáticas evaluadas en los exámenes de ingreso al nivel universitario; con el presente estudio, en cambio, se profundiza aún mas no solo en la ausencia de la competencia sino en la disimilitud de lo que se requiere como competencia matemática en un alumno de nuevo ingreso y lo que evalúan estas pruebas; se enriquece el estudio al comparar lo que se entiende en cada prueba estandarizada PISA, Planea y EXANI como competencia; y se compara con el estudio inicial de Rico (2007) que señala la importancia de ubicar las competencias matemáticas y buscar que los estudiantes los alcancen según su nivel educativo. Por ello, ubicado en este caso un estadio por niveles de pensamiento como lo muestra Cuevas et al. (2017), este marco sirve de base al análisis y al diseño del Pretest. 


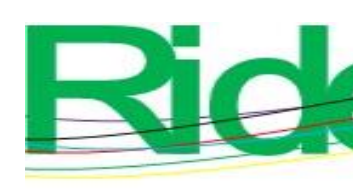

Revista Iberoamericana para la

Investigación y el Desarrollo Educativo

ISSN 2007 - 7467

\section{Conclusiones}

A partir de los resultados de las pruebas estandarizadas para medir las competencias matemáticas en alumnos de nivel medio superior, se observa una similitud de alcances. Por ejemplo, conforme a los resultados de Planea 2015 para el caso de Matemáticas, el puntaje promedio nacional fue de 498 y en 2017 de 500 puntos, donde cerca de las dos terceras partes de los estudiantes se concentra en el nivel más bajo del desempeño. En los resultados 2016 de Planea MS para el municipio de Valle de Chalco, donde pertenecen las instituciones de los sujetos de la muestra, se tiene que $72 \%$ se encuentra en nivel 1, 20\% en nivel 2, $8 \%$ en nivel 3 y ninguno en nivel 4; lo mismo sucede si revisamos los niveles nacionales: $49.2 \%$ está en nivel 1, 30\% en nivel 2, $14.4 \%$ en nivel 3 y $6.3 \%$ en nivel 4. Algo similar se encuentra con PISA 2015, donde el desempeño de México se encuentra por debajo del promedio OCDE en Matemáticas (408 puntos) y menos de $1 \%$ de los estudiantes en México logran alcanzar niveles de competencia de excelencia (nivel 5 y 6, de 607 a 688 puntos). Estos resultados son confirmados por el EXANI II, donde la gran mayoría apenas y aprueba las competencias matemáticas. Todos estos instrumentos confirman lo que muchas investigaciones y diferentes medios de noticias publican en los últimos años, que los estudiantes mexicanos no adquieren las competencias necesarias para su desempeño en matemáticas.

Sin embargo, como profesor se requiere un análisis más profundo, ya que en los modelos educativos actuales se parte de un nivel del alumnado para llegar a una competencia mayor, por lo que la confiabilidad de los resultados del diagnóstico es de suma importancia. Ante esta enorme responsabilidad muchos profesores que imparten las asignaturas del primer semestre universitario acaban dando contenidos de aritmética en el curso de algebra, de algebra y aritmética en el de geometría analítica, y de todas las asignaturas en las de cálculo diferencial; lo que obstaculiza abordar los contenidos propios de cada materia. Así, en este trabajo se retomaron de las diferentes teorías de evaluación los indicadores de pensamiento aritmético, pensamiento algebraico y pensamiento funcional (Cuevas y Delgado, 2016) para analizar el tipo de razonamiento verbal, lógico matemático y funcional de los estudiantes y buscar correlacionar la información que aportan todos los instrumentos mencionados.

Para ello, se diseñó el instrumento denominado Pretest que contempla el conocimiento matemático inicial (prerrequisitos) que marcan los programas de estudio para alumnos de nuevo ingreso a la carrera de Ingeniería en Computación del Centro Universitario Vale de 

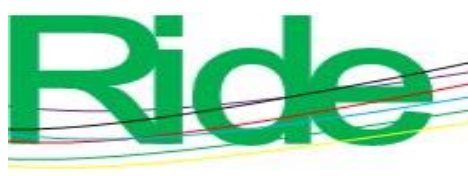

Revista Iberoamericana para la

Investigación y el Desarrollo Educativo

ISSN $2007-7467$

Chalco, de la Universidad Autónoma del Estado de México, en el periodo 2017, y se correlacionaron los datos con las competencias matemáticas consideradas en las pruebas estandarizadas (PISA, Planea y EXANI), se evaluaron conocimientos básicos de aritmética, algebra, geometría analítica y cálculo diferencial en $50 \%$ de la población de alumnos de ingeniería. Reflejando que en particular que el EXANI no considera los contenidos para determinar la competencia matemática de alumnos de nuevo ingreso a esta carrera.

La correlación de Pearson y la comparación de medias por t de student muestran la falta de concordancia en contenidos $\mathrm{y}$, por tanto, del alcance de cada prueba. En todos los instrumentos se obtiene un bajo nivel operativo de los estudiantes. Si se parte de la posición de que estas evaluaciones sirven para mejorar el desempeño de los estudiantes, resultan valiosos como punto de partida para incentivar procesos y revisar conceptos con los alumnos que los lleven a alcanzar las competencias matemáticas descritas. En ese sentido, se recomiendan actividades didácticas empleando tecnología diseñadas con la intención de favorecer el aprendizaje significativo y conceptual, como parte de una experiencia por parte del Cuerpo Académico de Cómputo Aplicado, del área de Tecnología Educativa, del Centro Universitario Valle de Chalco, que viene desarrollando desde hace 10 años (Soberanes, Martínez y Castillo, 2016), y así fomentar un desempeño más acorde a las características de la carrera profesional. 


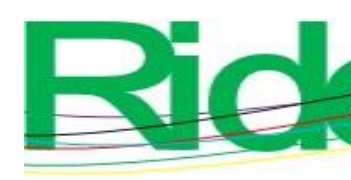

Revista Iberoamericana para la

Investigación y el Desarrollo Educativo

ISSN 2007 - 7467

\section{Bibliografía}

Aristegui, A. (1999). Indeterminación de la traducción radical, constructivismo y psicoterapia. Cinta de Moebio, 6, 1-18.

Aparicio, J. A., Hoyos, O., \& Niebles, R. (2004). Las concepciones implícitas de los profesores sobre el aprendizaje, Psicología desde el Caribe, 13, 144-168.

Bruno, A., Noda, M., Aguilar, R., González, C., Moreno, L., \& Muñoz, V. (2006). Análisis de un Tutorial Inteligente sobre conceptos lógico-matemáticos en alumnos con síndrome de Down. Revista latinoamericana de Investigación Matemática, 9(2), 211-226.

Centro Nacional de Evaluación para la Educación Superior (2016). Planea MS 2016. Manual para usuarios. CENEVAL.

Centro Nacional de Evaluación para la Educación Superior. (2017). Guía EXANI II. Examen Nacional de Ingreso a la Educación Superior. CENEVAL.

Cuevas, C. \& Pluvinage, F. (2017). Revisando la noción de función real. El Cálculo y su Enseñanza, Enseñanza de las Ciencias y la Matemática, 8, 31-48.

Filloy, E., Puig, L., \& Rojano, T. (2008). El estudio teórico local del desarrollo de competencias algebraicas. Enseñanza de las Ciencias, 25(3), 327-342.

García, V. J. (2002). La generación de nuevo conocimiento a partir de los errores. Revista de Economía, 11(1), 2-19.

García, J. (2014). Ingeniería, Matemáticas y Competencias. Revista Electrónica Actualidades Investigativas en Educación, 14 (1), 1-29.

Instituto Nacional para la Evaluación de la Educación. (2012). Pisa en el Aula: Matemáticas. INEE.

Instituto Nacional para la Evaluación de la Educación. (2015). Panorama educativo de México 2015. Educación básica y Media superior. INEE.

Instituto Nacional para la Evaluación de la Educación. (2016). Plan Nacional para la Evaluación de los Aprendizajes (PLANEA). INEE.

Instituto Nacional para la Evaluación de la Educación. (2017). Plan Nacional para la Evaluación de los Aprendizajes (Planea). Resultados nacionales 2017 Educación Media Superior. INEE.

Kjeldsen, T.H. \& Petersen, H. P. (2014). Brindging History of the Concept of Function with Learning of Mathematics: Students 'Meta-Discursive Rules, Concept Formation and Historical Awareness. Science \& Education, 23, 29-45. 


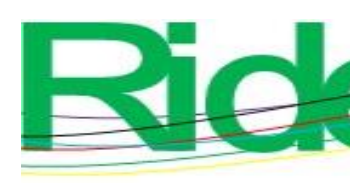

Revista Iberoamericana para la Investigación y el Desarrollo Educativo ISSN $2007-7467$

Lagrange, J.B. (2014). A Functional Perspective on the Teaching of Algebra: Current Challenges and the Contribution of Technology. The International Journal for Technology in Mathematics Education, 21(1), 3-10.

Larrazolo, N., Backhoff, E. \& Tirado, F. 2013. Habilidades de razonamiento matemático de estudiantes de Educación Media Superior en México. Revista Mexicana de Investigación Educativa, 18(59), 1137-1163.

Lejter, J. (1990). Instrucción y aprendizaje significativo. Caracas: Universidad Pedagógica Libertador.

Martínez, M., Soberanes, A. \& Castillo, J. (octubre, 2016). Análisis comparativo de pretest y postest para determinar los prerrequisitos matemáticos en un curso de Cálculo diferencial en el primer año del nivel superior. Memorias del 1er Congreso Nacional de Matemáticas, su Enseñanza y Aprendizaje, Escuela Normal Superior de México.

Mouhayar, R.E. \& Jurdak, M. (2016). Variation of Student Numerical and Figural Reasoning Approaches by Pattern Generalization Type, Strategy Use and Grade Level. International Journal of Mathematical Education in Science and Technology, 47(2), 197-215.

Narváez, R. M. (2005). Aprendizaje auto dirigido y desempeño académico. Tiempo de Educar, 6(11), 115-146.

National Council of Teachers of Mathematics. (1989). Curriculum and Evaluation Standards for School Mathematics. Reston, VA: The Author.

Organización para la Cooperación y el Desarrollo Económico. (2009). El programa PISA de la OCDE. Qué es y para qué sirve. OCDE.

Organización para la Cooperación y el Desarrollo Económico. (2015). Programa para la Evaluación Internacional de Alumnos (PISA) 2015 Resultados. OCDE. Recuperado de: https://www.oecd.org/pisa/PISA-2015-Mexico-ESP.pdf.

Organización para la Cooperación y el Desarrollo Económico. (2017). Panorama educativo de la educación 2017. OCDE-INEE.

Padilla, M. (2003). La comprensión del cerebro. Hacia una nueva ciencia del aprendizaje. Perfiles Educativos, 27(109), 224-227.

Padilla, V., \& López, E. (2006). Implementación de una red neural para estilos cognitivos y de aprendizaje: Implicaciones Educativas. Enseñanza e Investigación en Psicología, 11(2), 239-254. 

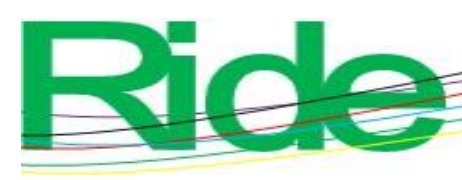

Revista Iberoamericana para la

Investigación y el Desarrollo Educativo

ISSN $2007-7467$

Pluvinage, F. \& Cuevas, A. (2006). Un acercamiento didáctico a la noción de función. En Eugenio Filloy (ed.). Matemática Educativa, treinta años: Una mirada fugaz, una mirada externa y comprensiva, una mirada actual (pp. 141-167). México: Santillana.

Rico, L. (2007). La competencia matemática en PISA. PNA, 1(2), 47-66.

Seeley, C. 2004. NCTM News Bulletin, September. Recuperado de http://www.nctm.org/News-and-Calendar/Messages-from-thePresident/Archive/Cathy-Seeley/A-Journey-in-Algebraic-Thinking/.

Secretaria de Educación Pública. (2008). Competencias genéricas y Perfil del egresado de la Educación Media Superior. Subsecretaria de Educación Media Superior de la Secretaria de Educación Pública de México.

Secretaria de Educación Pública. (2017). Diferencias entre las pruebas de Planea y EXANI II. SEP.

Soberanes, A., Martínez, M, \& Juárez, C. (2016). Recursos digitales como apoyo en la enseñanza matemática en educación superior, Revista Programación Matemática y Software, 8(2), 53-59.

Viera, T. (2003). El aprendizaje verbal significativo de Ausubel. Universidades, 26, 37-43.

Villalón, M., Medina, M., \& Bravo, M. (2015). Competencias matemáticas previas de los estudiantes de Ingeniería. Revista Electrónica ANFEI Digital, 1(2), 1-10. 
Revista Iberoamericana para la

Investigación y el Desarrollo Educativo

ISSN 2007 - 7467

\begin{tabular}{|c|c|}
\hline Rol de Contribución & Autor (es) \\
\hline Conceptualización & Magally, Anabelem y Juan Manuel «igual» \\
\hline Metodología & Anabelem «principal», Magally y Juan Manuel «que apoyan». \\
\hline Software & Juan Manuel «principal», Anabelem y Magally «que apoyan». \\
\hline Validación & Magally, Anabelem y Juan Manuel «igual» \\
\hline Análisis Formal & Magally, Anabelem y Juan Manuel «igual» \\
\hline Investigación & Magally «principal», Anabelem y Juan Manuel «que apoyan». \\
\hline Recursos & Anabelem «principal», Magally y Juan Manuel «que apoyan». \\
\hline Curación de datos & Juan Manuel «principal», Anabelem y Magally «que apoyan». \\
\hline $\begin{array}{l}\text { Escritura - Preparación del } \\
\text { borrador original }\end{array}$ & Magally, Anabelem y Juan Manuel «igual» \\
\hline Escritura - Revisión y edición & Anabelem, Magally y Juan Manuel «igual» \\
\hline Visualización & Anabelem «principal», Magally y Juan Manuel «que apoyan». \\
\hline Supervisión & Magally «principal», Anabelem y Juan Manuel «que apoyan». \\
\hline Administración de Proyectos & Anabelem, Magally y Juan Manuel «igual» \\
\hline Adquisición de fondos & Anabelem «principal», Magally y Juan Manuel «que apoyan». \\
\hline
\end{tabular}

